\title{
Which Side-Bending X-ray Position is Better to Evaluate the Preoperative Curve Flexibility in Adolescent Idiopathic Scoliosis Patients, Supine or Prone?
}

\author{
Hirofumi Bekki ${ }^{1}$, Katsumi Harimaya ${ }^{1}$, Yoshihiro Matsumoto ${ }^{1}$, Kenichi Kawaguchi ${ }^{1}$, \\ Mitsumasa Hayashida ${ }^{1}$, Seiji Okada ${ }^{1}$, Toshio Doi ${ }^{2}$, Yasuharu Nakashima ${ }^{1}$ \\ ${ }^{1}$ Department of Orthopaedic Surgery, Graduate School of Medical Sciences, Kyushu University, Fukuoka, Japan \\ ${ }^{2}$ Department of Orthopaedic Surgery, Kyushu University Beppu Hospital, Oita, Japan
}

\section{Study Design: Prospective cohort study.}

Purpose: The present study aimed to evaluate the difference in the preoperative curve flexibility between the supine and prone positions in patients with adolescent idiopathic scoliosis (AIS).

Overview of Literature: In AIS, a side-bending view is necessary to differentiate a structural curve from a nonstructural curve using the Lenke classification system. However, there are no published studies about which position, supine or prone, is more effective when evaluating preoperative curve flexibility using side-bending X-ray images in AIS patients.

Methods: Radiographs were analyzed for 32 AIS patients (26 females, six males) who underwent posterior correction and fusion of their main thoracic (MT) curves. Cobb angles of MT, proximal thoracic (PT), and thoracolumbar/lumbar (TL/L) curves were measured preoperatively using upright, supine (anteroposterior and side-bending), and prone (posteroanterior and side-bending) X-rays.

Results: The average Cobb angles of PT, MT, and TL/L curves on preoperative upright/supine/prone X-rays were $29.1^{\circ} / 26.7^{\circ} / 26.6^{\circ}$, $60.7^{\circ} / 48.5^{\circ} / 48.2^{\circ}$, and $41.0^{\circ} / 32.6^{\circ} / 33.1^{\circ}$, respectively. The average Cobb angles of PT, MT, and TL/L curves on supine/prone sidebending X-rays were $19.2^{\circ} / 20.3^{\circ}, 36.3^{\circ} / 36.4^{\circ}$, and $13.9^{\circ} / 15.7^{\circ}$, respectively. The flexibility rates of PT, MT, and TL/L curves in supine/ prone positions were $35.3 \% / 32.5 \%, 40.6 \% / 40.2 \%$, and $71.7 \% / 68.2 \%$, respectively. Comparing flexibility rates in the prone position with those in the supine position in each case, the average ratios of PT, MT, and TL/L curves were found to be 1.0, 1.0, and 0.9, respectively. There were no statistically significant differences between supine and prone side-bending X-ray measurements. However, the Lenke classification in six of 32 patients (18.8\%) differed between supine and prone positions because the TL/L curve in the supine position was slightly more flexible than in the prone position.

Conclusions: Supine side-bending films may be suitable for the evaluation of preoperative curve flexibility in AIS, especially for lumbar modifier C.

Keywords: Scoliosis; X-rays; Supine position; Prone position

\footnotetext{
Received Aug 16, 2017; Revised Nov 30, 2017; Accepted Dec 25, 2017

Corresponding author: Katsumi Harimaya

Department of Orthopaedic Surgery, Graduate School of Medical Sciences, Kyushu University, 3-1-1 Maidashi, Higashi-ku, Fukuoka, 812-8582, Japan

Tel: +81-92-642-5488, Fax: +81-92-642-5507, E-mail: harimaya@ortho.med.kyushu-u.ac.jp
} 


\section{Introduction}

Precise preoperative assessment of curve flexibility in patients with adolescent idiopathic scoliosis (AIS) is important to determine the necessary level of corrective scoliosis surgery [1]. Although side-bending X-rays are the gold standard to evaluate curve flexibility because they remove the effects of gravity, other methods have been developed and used to evaluate curve flexibility. One of these methods includes the use of fulcrum-bending radiographs, which reportedly demonstrate good flexibility while also being easy to perform [2]. The other popular method includes the use of push-prone radiographs, where the physician applies manual pressure to the apices of the curve while images are being taken [3]. However, the side-bending view is still necessary in AIS to distinguish a structural curve from a nonstructural curve in the Lenke classification system [4].

AIS patients are exposed to potentially damaging Xrays during childhood and adolescence. To reduce the risk from this exposure, the posteroanterior (PA) projection is recommended when taking X-rays [5]. To the best of our knowledge, there is no report regarding whether the supine or prone position for side-bending X-ray images is more effective when evaluating the preoperative curve flexibility in AIS patients. This study aimed to evaluate the difference in preoperative curve flexibility between the prone and supine positions in AIS patients.

\section{Materials and Methods}

This study included 32 patients who underwent posterior correction and fusion of their main thoracic (MT) curve to treat AIS. The curves were evaluated as previously described [6]. Briefly, curves were classified into proximal thoracic (PT), MT, and thoracolumbar/lumbar (TL/L), depending on the location of the apex of the curve and using the Cobb method with upright position X-rays. The patients included 26 females and six males with an average age of 15.1 years (range, 11-21 years) at the time of surgery. Long-cassette $(91.44 \mathrm{~cm}) \mathrm{X}$-rays of the spine included preoperative upright PA, supine anteroposterior, and side-bending as well as prone PA and side-bending positioning. As described in a previous study, supine sidebending radiographs were obtained by maximal passive bending while keeping the neck and trunk in neutral rotation [7]. The head of the patient was turned to the bend- ing direction during the prone position. Two patients had PT curves $<10^{\circ}$ in the upright X-ray; hence, both patients were excluded from this study. Flexibility rate (FR) was calculated using the following formula: $F R \%=$ (upright angle-side-bending angle)/upright angle $\times 100$ [6-8]. The Cobb angles and FR were compared between the supine and prone positions. The measurements were obtained by a board-certified spine surgeon (K.H.) and a boardcertified orthopedic surgeon (H.B.). Measurements were performed 2 times on different days. Intra- and interobserver reliability was evaluated using the intraclass correlation coefficient by R software ver. 2.15.2 (R Core Team, https://www.r-project.org/). Intraobserver reliability was 0.98 , and interobserver reliability was 0.94 . The average of the two measurements was used for statistical analysis.

Student $t$-test was used to analyze the difference between the two positions, and Pearson's correlation coefficient $(\mathrm{R})$ was used to evaluate the correlation between the two position. Non-parametric analysis followed by Mann-Whitney $U$-test was used to analyze radiographic data between the two positions. All $p$-values of $<0.05$ were considered statistically significant.

The Institutional Review Board at the Kyushu University approved this retrospective study (IRB permission code, 26-112).

\section{Results}

\section{Cobb angle}

The average Cobb angle for each body position is shown in Table 1. The average Cobb angles of the PT, MT, and $\mathrm{TL} / \mathrm{L}$ curves in the upright position were $29.1^{\circ} \pm 11.0^{\circ}$, $60.7^{\circ} \pm 12.1^{\circ}$, and $41.0^{\circ} \pm 14.3^{\circ}$, respectively. The average Cobb angles of the PT, MT, and TL/L curves in the supine position were $26.7^{\circ} \pm 9.9^{\circ}, 48.5^{\circ} \pm 9.8^{\circ}$, and $32.6^{\circ} \pm 12.1^{\circ}$, respectively. The average Cobb angles of the PT, MT, and $\mathrm{TL} / \mathrm{L}$ curves in the supine side-bending position were $19.2^{\circ} \pm 9.6^{\circ}, 36.3^{\circ} \pm 10.5^{\circ}$, and $13.9^{\circ} \pm 13.9^{\circ}$, respectively. Alternatively, the average Cobb angles of the PT, MT, and $\mathrm{TL} / \mathrm{L}$ curves in the prone position were $26.6^{\circ} \pm 10.2^{\circ}$, $48.2^{\circ} \pm 9.2^{\circ}$, and $33.1^{\circ} \pm 13.7^{\circ}$, respectively. The average Cobb angles of the PT, MT, and TL/L curves in the prone side-bending position were $20.3^{\circ} \pm 11.1^{\circ}, 36.4^{\circ} \pm 10.6^{\circ}$, and $15.7^{\circ} \pm 16.3^{\circ}$, respectively. There was no statistically significant difference between the supine and prone positions $(p>0.05)$. The correlation between Cobb angles in the su- 
Table 1. Cobb angles for body positions

\begin{tabular}{|c|c|c|c|c|c|}
\hline Curve & Upright & Supine & Prone & Supine side-bending & Prone side-bending \\
\hline \multicolumn{6}{|c|}{ proximal thoracic $\left(^{\circ}\right)(n=30)$} \\
\hline Mean $\pm S D$ & $29.1 \pm 11.0$ & $26.7 \pm 9.9$ & $26.6 \pm 10.2$ & $19.2 \pm 9.6$ & $20.3 \pm 11.1$ \\
\hline Minimum & 10.0 & 9.0 & 8.0 & 7.5 & 5.0 \\
\hline Maximum & 56.0 & 52.5 & 54.0 & 46.5 & 54.0 \\
\hline \multicolumn{6}{|c|}{ main thoracic $\left({ }^{\circ}\right)(n=32)$} \\
\hline Mean $\pm S D$ & $60.7 \pm 12.1$ & $48.5 \pm 9.8$ & $48.2 \pm 9.2$ & $36.3 \pm 10.5$ & $36.4 \pm 10.6$ \\
\hline Minimum & 46.0 & 34.5 & 34.5 & 14.0 & 17.0 \\
\hline Maximum & 91.5 & 68.5 & 73.5 & 60.0 & 61.0 \\
\hline \multicolumn{6}{|c|}{ thoracolumbar/lumbar $\left({ }^{\circ}\right)(n=32)$} \\
\hline Mean $\pm S D$ & $41.0 \pm 14.3$ & $32.6 \pm 12.1$ & $33.1 \pm 13.7$ & $13.9 \pm 13.9$ & $15.7 \pm 16.3$ \\
\hline Minimum & 22.5 & 14.0 & 3.5 & -15.0 & -16.5 \\
\hline Maximum & 78.5 & 62.0 & 71.5 & 46.0 & 58.0 \\
\hline
\end{tabular}

SD, standard deviation.
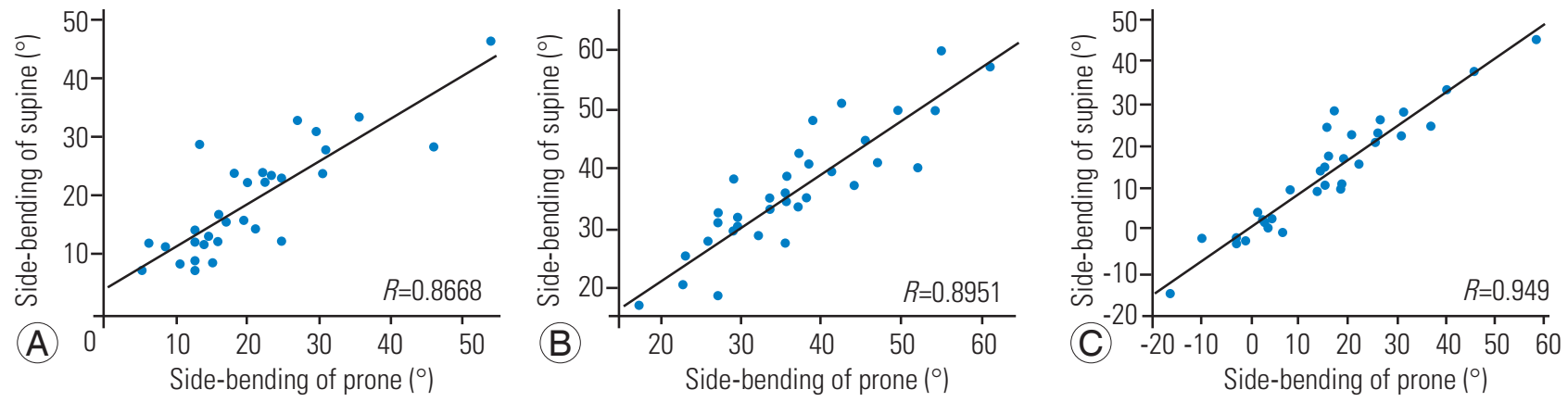

Fig. 1. There are significant correlations between the Cobb angles of any curve in both the supine and prone side-bending positions ( $p<0.01)$. (A) Proximal thoracic. (B) Main thoracic. (C) Thoracolumbar/lumbar. R, correlation coefficient.

Table 2. Flexibility rates for supine and prone positions

\begin{tabular}{|c|c|c|c|}
\hline Curve & Supine & Prone & $p$-value \\
\hline \multicolumn{4}{|c|}{ Proximal thoracic $(\%)(n=30)$} \\
\hline Mean $\pm S D$ & $35.3 \pm 15.5$ & $32.5 \pm 16.4$ & 0.5172 \\
\hline Minimum & 1.7 & 3.6 & \\
\hline Maximum & 64.6 & 61.3 & \\
\hline \multicolumn{4}{|c|}{ Main thoracic $(\%)(n=32)$} \\
\hline Mean $\pm S D$ & $40.6 \pm 11.1$ & $40.2 \pm 11.5$ & 0.8106 \\
\hline Minimum & 23.1 & 20.6 & \\
\hline Maximum & 72.8 & 67.0 & \\
\hline \multicolumn{4}{|c|}{ Thoracolumbar/lumbar (\%) (n=32) } \\
\hline Mean $\pm S D$ & $71.7 \pm 28.8$ & $68.2 \pm 34.8$ & 0.5682 \\
\hline Minimum & 31.3 & 2.7 & \\
\hline Maximum & 157.7 & 163.5 & \\
\hline
\end{tabular}

SD, standard deviation. 

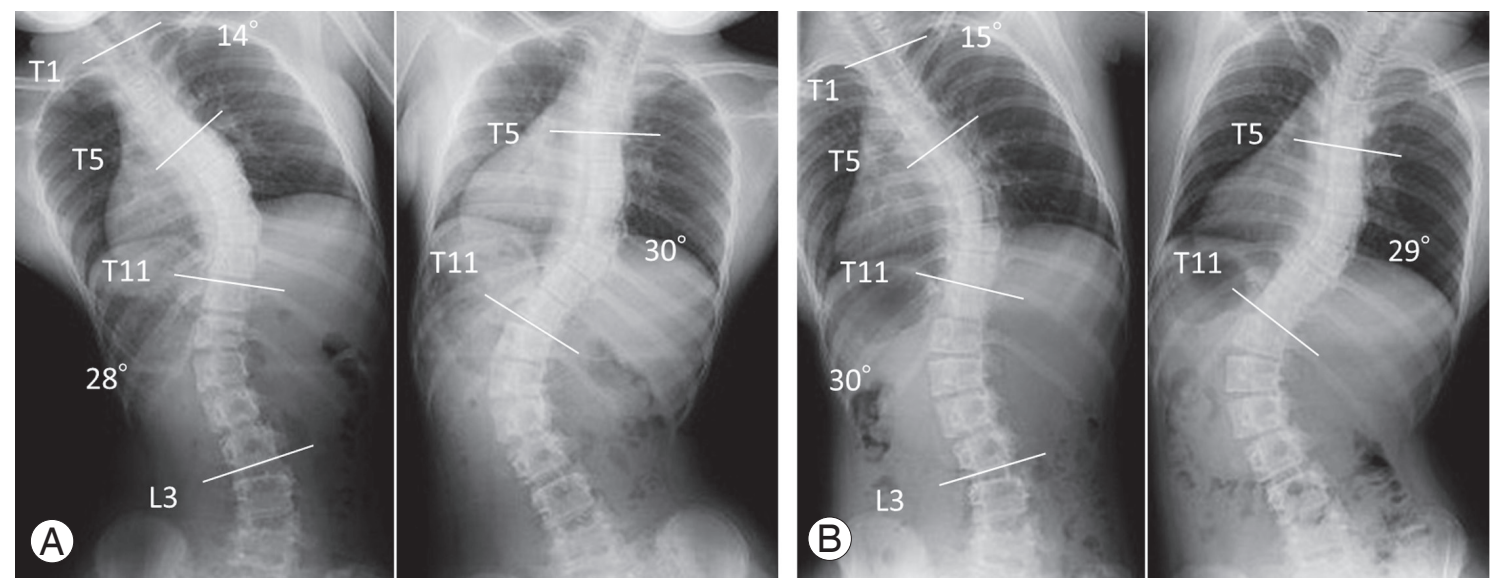

Fig. 2. A representative case without a difference in Cobb angles for supine and prone positions. Cobb angles in side-bending positions were almost similar between the supine and prone positions. (A) Supine side-bending. (B) Prone side-bending.
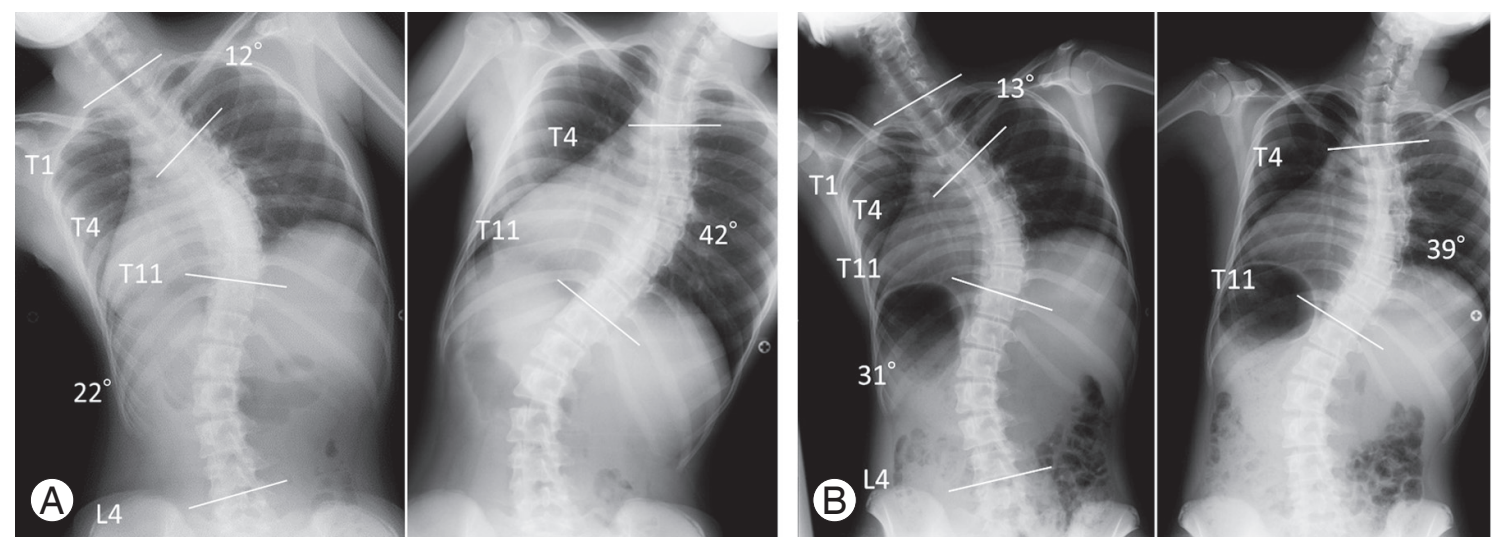

Fig. 3. A representative case with a difference in Cobb angles for supine and prone positions. The side-bending angles of thoracolumbar/lumbar curves in the supine and prone positions were $22^{\circ}$ and $31^{\circ}$, respectively. The Lenke classification using supine sidebending $\mathrm{X}$-rays was type $1 \mathrm{C}$, and the classification was type $3 \mathrm{C}$ when using prone side-bending $\mathrm{X}$-rays. (A) Supine side-bending. (B) Prone side-bending.

pine and prone side-bending positions is illustrated in Fig. 1. The correlation coefficients (R) of the PT, MT, and TL/L curves were $0.837,0.895$, and 0.949 , respectively $(p<0.01)$. A representative case without a difference in Cobb angles for supine and prone positions is illustrated in Fig. 2. Sidebending angles were approximately the same when comparing the prone and supine positions.

\section{Flexibility rate}

The average FRs are shown in Table 2. The average FRs of the PT, MT, and TL/L curves in the supine position were $35.3 \% \pm 15.5 \%, 40.6 \% \pm 11.1 \%$, and $71.7 \% \pm 28.8 \%$, respectively. The average FRs of the PT, MT, and TL/L curves in the prone position were $32.5 \% \pm 16.4 \%, 40.2 \% \pm 11.5 \%$, and $68.2 \% \pm 34.8 \%$, respectively. In each case, when the prone- position FR was compared with the supine-position FR, the average ratios of the $\mathrm{PT}, \mathrm{MT}$, and $\mathrm{TL} / \mathrm{L}$ curves were $0.973 \pm 0.593,1.007 \pm 0.213$, and $0.926 \pm 0.256$, respectively (Table 3 ). There was no statistically significant difference in the FR in the prone and supine positions $(p>0.05)$.

\section{Lenke classification}

As listed in Table 4, the Lenke classification in six of the $32(18.8 \%)$ patients differed between the supine and prone positions. Four curves that were classified as Lenke 1C in the supine position were classified as Lenke $3 \mathrm{C}$ in the prone position. One curve that was classified as Lenke $2 \mathrm{~B}$ in the supine position was considered as Lenke $1 \mathrm{~B}$ in the prone position. One curve classified as Lenke $3 \mathrm{C}$ in the supine position was considered as Lenke $4 \mathrm{C}$ in the 
Table 3. Flexibility rates for prone and supine positions

\begin{tabular}{lc} 
Curve & Flexibility rate \\
Proximal thoracic side-bending $(\mathrm{n}=30)$ & \\
\hline Mean \pm SD & $0.973 \pm 0.593$ \\
\hline Minimum & 0.167 \\
\hline Maximum & 2.714 \\
\hline Main thoracic side-bending $(\mathrm{n}=32)$ & \\
\hline Mean $\pm S D$ & $1.007 \pm 0.213$ \\
\hline Minimum & 0.540 \\
\hline Maximum & 1.613 \\
\hline Thoracolumbar/lumbar side-bending $(\mathrm{n}=32)$ & \\
\hline Mean $\pm S D$ & $0.926 \pm 0.256$ \\
\hline Minimum & 0.077 \\
\hline Maximum & 1.409 \\
\hline
\end{tabular}

SD, standard deviation. prone position. X-rays of case 5 in Table 4 are illustrated in Fig. 3. For the TL/L curve, the average side-bending angles of supine and prone positions were $22.5^{\circ}$ and $30.5^{\circ}$, respectively. The Lenke classification in this case differed between the supine and prone positions.

In total, the preoperative Lenke classifications using supine side-bending $\mathrm{X}$-rays were type 1 in 20 of the patients (lumbar modifier A in 13, B in two, and C in five). Overall, six patients were type 2 (lumbar modifier A in five, $B$ in one), three were type 3 , one was type 4 , and two patients were type 6 . Alternatively, the Lenke classifications using prone side-bending $\mathrm{X}$-rays were type 1 in 17 patients (lumbar modifier A in 13, B in three, and C in one), type 2 in five patients (lumbar modifier A in five), type 3 in six patients, type 4 in two patients, and type 6 in two patients.

Table 4. Lenke classification of six cases for supine and prone positions

\begin{tabular}{|c|c|c|c|c|c|}
\hline Variable & Curve & $\begin{array}{c}\text { Side-bending angle } \\
\left.\text { (supine, }{ }^{\circ}\right)\end{array}$ & $\begin{array}{c}\text { Lenke classification } \\
\text { (supine) }\end{array}$ & $\begin{array}{l}\text { Side-bending angle } \\
\left(\text { prone }^{\circ}\right)\end{array}$ & $\begin{array}{c}\text { Lenke classification } \\
\text { (prone) }\end{array}$ \\
\hline \multicolumn{6}{|c|}{ Case 1: $16 \mathrm{yr}, \mathrm{F}$} \\
\hline PT & T1-T5 & 22.5 & & 22.5 & \\
\hline MT & T5-T11 & 35 & $1 \mathrm{C}$ & 35.5 & $3 C$ \\
\hline $\mathrm{TL} / \mathrm{L}$ & T11-L3 & 21 & & 25.5 & \\
\hline \multicolumn{6}{|c|}{ Case 2: 14 yr, F } \\
\hline PT & T1-T5 & 9 & & 12.5 & \\
\hline MT & T5-T11 & 28 & $1 \mathrm{C}$ & 35.5 & $3 C$ \\
\hline $\mathrm{TL} / \mathrm{L}$ & $\mathrm{T} 11-\mathrm{L} 3$ & 24.5 & & 36.5 & \\
\hline \multicolumn{6}{|c|}{ Case 3: $15 \mathrm{yr}, \mathrm{F}$} \\
\hline $\mathrm{PT}$ & $\mathrm{T} 1-\mathrm{T} 6$ & 29 & & 13 & \\
\hline MT & T6-T12 & 29 & $2 B$ & 32 & 1B \\
\hline $\mathrm{TL} / \mathrm{L}$ & T12-L5 & 17.5 & & 16 & \\
\hline \multicolumn{6}{|c|}{ Case 4: 15 yr, M } \\
\hline PT & T1-T5 & 24 & & 30.5 & \\
\hline MT & T5-T11 & 37.5 & $3 \mathrm{C}$ & 44 & $4 C$ \\
\hline $\mathrm{TL} / \mathrm{L}$ & T11-L3 & 33.5 & & 40 & \\
\hline \multicolumn{6}{|c|}{ Case 5: 15 yr, F } \\
\hline PT & $\mathrm{T} 1-\mathrm{T} 4$ & 12 & & 14 & \\
\hline MT & $\mathrm{T} 4-\mathrm{T} 11$ & 41 & $1 \mathrm{C}$ & 38.5 & $3 C$ \\
\hline $\mathrm{TL} / \mathrm{L}$ & T11-L4 & 22.5 & & 30.5 & \\
\hline \multicolumn{6}{|c|}{ Case 6: 13 yr, F } \\
\hline PT & T1-T5 & 24 & & 22 & \\
\hline MT & T5-T11 & 30.5 & $1 \mathrm{C}$ & 29.5 & $3 C$ \\
\hline $\mathrm{TL} / \mathrm{L}$ & T11-L3 & 23 & & 26 & \\
\hline
\end{tabular}

F, female; $M$, male; $P T$, proximal thoracic; $M T$, main thoracic; $T L / L$, thoracolumbar/lumbar. 
For the cases with lumbar modifier $C(n=11)$, there was no significant difference between supine side-bending and prone side-bending positions in the Cobb angles (average, supine $27.9^{\circ}$ versus prone $32.6^{\circ}, p=0.38$ ) and FR of the TL/ L curve (average: supine 49.3 versus prone $43.8, p=0.23$ ). However, this comparison indicated that there is slightly more flexibility in the supine position than in the prone position.

\section{Discussion}

The Lenke classification is the most common method to evaluate AIS worldwide [1]. The criteria of the structural minor curve for AIS is defined as a side-bending Cobb angle of $>25^{\circ}$ or a kyphosis angle of $>20^{\circ}$. Some methods such as push-prone and fulcrum-bending have been reported to achieve maximal preoperative correction during assessment [3,7-9]. However, side-bending X-rays are still very effective when evaluating the preoperative flexibility and structure of curves [4].

The radiation exposure in AIS patients during $\mathrm{X}$-rays can be problematic. AIS patients who are exposed to multiple radiographic examinations are at an increased risk for breast cancer [8-10]. To minimize the risk from this exposure, PA projection is recommended. The use of PA projection results in a $95 \%$ reduction in exposure to the lens of the eye during intracranial tomography and $>90 \%$ reduction in exposure to the thyroid, sternum, and breasts during scoliosis radiography [5]. To assess which position is suitable for the evaluation of preoperative curve flexibility in AIS patients, the difference in X-ray parameters between the supine and prone positions were investigated. It is concluded that there is no statistically significant difference in the Cobb angle and FR between the supine and prone positions. These results indicated that both positions are suitable when evaluating the preoperative curve flexibility in AIS patients.

Interestingly, the Lenke classification in six of the 32 (18.2\%) patients is different for the supine and prone positions. In four of the six (66.6\%), curves with Lenke $1 C$ in the supine position are considered as Lenke $3 \mathrm{C}$ in the prone position. For lumbar modifier $\mathrm{C}$, it is possible that the side-bending Cobb angles of the TL/L curve are influenced by the body position. It is also possible that there is a difference in the side-bending Cobb angles and FR of the TL/L curve between the supine and prone positions. Although not statistically significant, there is slightly more flexibility in the supine position than in the prone position among cases with lumbar modifier $\mathrm{C}$. These results indicated that supine side-bending $\mathrm{X}$-rays may be more suitable when evaluating preoperative curve flexibility, especially for lumbar modifier C.

Side-bending X-rays are difficult to standardize, and some studies have reported that side-bending positions have a low reproducibility rate with varying results due to the muscular effort of the patient $[11,12]$. In this study, the data for side-bending Cobb angles of PT curves show more variability than those for MT and TL/L curves. Considering that PT curves are often stiffer than MT curves, the low reproducibility of the side-bending X-rays may be reflected in PT curves [13]. Another possibility is that the head turns to the side in the prone position and the angles of PT curves in the prone position may be dependent on the cooperation of patients. However, TL/L curves do not seem to be dependent on head positioning because there is a strong correlation between Cobb angles in the supine and prone positions (Fig. 1). Our results show that there is slightly more flexibility in the supine position than in the prone position among cases with lumbar modifier C. Although more cases and further studies are needed, the results of this study may shed light on which position is better to evaluate the flexibility of TL/L curves.

\section{Conclusions}

Considering the need to reduce radiation exposure, prone side-bending X-rays are generally recommended. However, prone side-bending positions have a low reproducibility rate for PT curves, and there is more flexibility in the supine position than in the prone position among cases with lumbar modifier $\mathrm{C}$. The supine side-bending X-rays may be suitable for the evaluation of preoperative curve flexibility, especially for lumbar modifier C.

\section{Conflict of Interest}

No potential conflict of interest relevant to this article was reported.

\section{References}

1. Lenke LG, Betz RR, Harms J, et al. Adolescent idiopathic scoliosis: a new classification to determine extent of spinal arthrodesis. J Bone Joint Surg Am 
2001;83:1169-81.

2. Luk KD, Cheung KM, Lu DS, Leong JC. Assessment of scoliosis correction in relation to flexibility using the fulcrum bending correction index. Spine (Phila Pa 1976) 1998;23:2303-7.

3. Harfouch BF, Weinstein SL. Intraoperative pushprone test: a useful technique to determine the lowest instrumented vertebra in adolescent idiopathic scoliosis. J Spinal Disord Tech 2014;27:237-9.

4. Klepps SJ, Lenke LG, Bridwell KH, Bassett GS, Whorton J. Prospective comparison of flexibility radiographs in adolescent idiopathic scoliosis. Spine (Phila Pa 1976) 2001;26:E74-9.

5. Frank ED, Stears JG, Gray JE, Winkler NT, Hoffman $\mathrm{AD}$. Use of the posteroanterior projection: a method of reducing $\mathrm{x}$-ray exposure to specific radiosensitive organs. Radiol Technol 1983;54:343-7.

6. Lenke LG, Betz RR, Clements D, et al. Curve prevalence of a new classification of operative adolescent idiopathic scoliosis: does classification correlate with treatment? Spine (Phila Pa 1976) 2002;27:604-11.

7. Kwan MK, Chiu CK, Chan TS, Abd Gani SM, Tan $\mathrm{SH}$, Chan CY. Flexibility assessment of the unfused thoracic segments above the "potential upper instrumented vertebrae" using the supine side bending radiographs in Lenke 5 and 6 curves for adolescent idiopathic scoliosis patients. Spine J 2018;18:53-62.
8. Watanabe K, Kawakami N, Nishiwaki Y, et al. Traction versus supine side-bending radiographs in determining flexibility: what factors influence these techniques? Spine (Phila Pa 1976) 2007;32:2604-9.

9. Li J, Hwang S, Wang F, et al. An innovative fulcrumbending radiographical technique to assess curve flexibility in patients with adolescent idiopathic scoliosis. Spine (Phila Pa 1976) 2013;38:E1527-32.

10. Ronckers CM, Doody MM, Lonstein JE, Stovall M, Land CE. Multiple diagnostic X-rays for spine deformities and risk of breast cancer. Cancer Epidemiol Biomarkers Prev 2008;17:605-13.

11. Lamarre ME, Parent S, Labelle H, et al. Assessment of spinal flexibility in adolescent idiopathic scoliosis: suspension versus side-bending radiography. Spine (Phila Pa 1976) 2009;34:591-7.

12. Cheh G, Lenke LG, Lehman RA Jr, Kim YJ, Nunley $\mathrm{R}$, Bridwell KH. The reliability of preoperative supine radiographs to predict the amount of curve flexibility in adolescent idiopathic scoliosis. Spine (Phila Pa 1976) 2007;32:2668-72.

13. Kirk KL, Kuklo TR, Polly DW Jr. Traction versus side-bending radiographs: is the proximal thoracic curve the stiffer curve in double thoracic curves? Am J Orthop (Belle Mead NJ) 2003;32:284-8. 